\title{
Bell's inequality and entanglement in qubits
}

\author{
Po-Yao Chang, ${ }^{a}$ Su-Kuan $\mathrm{Chu}^{b}$ and Chen-Te $\mathrm{Ma}^{c, 1}$ \\ ${ }^{a}$ Center for Materials Theory, Rutgers University, \\ Piscataway, New Jersey, 08854, U.S.A. \\ ${ }^{b}$ Joint Quantum Institute and Joint Center for Quantum Information and Computer Science, \\ NIST/University of Maryland, \\ College Park, Maryland, 20742, U.S.A. \\ ${ }^{c}$ Department of Physics and Center for Theoretical Sciences, National Taiwan University, \\ Taipei 10617, Taiwan, R.O.C. \\ E-mail: pychang@physics.rutgers.edu, skchu@terpmail.umd.edu, \\ yefgst@gmail.com
}

ABSTRACT: We propose an alternative evaluation of quantum entanglement by measuring the maximum violation of the Bell's inequality without information of the reduced density matrix of a system. This proposal is demonstrated by bridging the maximum violation of the Bell's inequality and a concurrence of a pure state in an $n$-qubit system, in which one subsystem only contains one qubit and the state is a linear combination of two product states. We apply this relation to the ground states of four qubits in the Wen-Plaquette model and show that they are maximally entangled. A topological entanglement entropy of the Wen-Plaquette model could be obtained by relating the upper bound of the maximum violation of the Bell's inequality to the generalized concurrence of a pure state with respect to different bipartitions.

Keywords: Topological Field Theories, Topological States of Matter

ARXIV EPRINT: 1705.06444

\footnotetext{
${ }^{1}$ Corresponding author.
} 


\section{Contents}

1 Introduction 1

2 Entanglement and maximum violation $\quad 2$

3 Applications to the Wen-Plaquette model 5

$\begin{array}{llr}4 & \text { Outlook } & 7\end{array}$

$\begin{array}{lll}\text { A } & \text { Maximum violation of the six-qubit state } & 7\end{array}$

\section{Introduction}

Entanglement measurements provide a way to extract quantum information from manybody wave-functions [1]. The most significant measure of entanglement is given by the entanglement entropy, $S_{A}=-\operatorname{Tr} \rho_{A} \ln \rho_{A}$ with $\rho_{A}=\operatorname{Tr}_{\mathrm{B}} \rho$ being the reduced density matrix of the subsystem $A$, and $\rho$ being the density matrix of a Hilbert space $\mathbb{H}=\mathbb{H}_{A} \otimes \mathbb{H}_{B}$. In other words, the entanglement entropy characterizes the entanglement between two complementary subsystems $A$ and $B$. The entanglement entropy has been observed experimentally in a two-qubit system, but measuring the entanglement entropy for a higher-qubit system is still under development.

On the other hand, a qualitative detection of quantum entanglement could be performed experimentally by the observation of the violation of the Bell's inequality [2]. The original theorem, proposed by John S. Bell [3], states that correlations between the outcomes of different measurements of two separated particles must satisfy the inequality under local realism. The violation of the constraints (the Bell's inequality) indicates the quantum effect of correlations or "entangledness" in quantum systems, which could be presented in two-qubit systems theoretically [4]. Although the violation of the Bell's inequality may not reveal the general structure of entanglement of a quantum state, the relation between the entanglement, measured in terms of the concurrence [5], and the violation of the Bell's inequality was shown in two-qubit systems [6,7]. The generalization for higher-qubit systems is still unclear.

In this letter, we discuss relations between the maximum violation of the Bell's inequality of an $n$-qubit Bell's operator [8] and the concurrence of a pure state when the $i$-th qubit operators in the Bell's operator are $\mathbf{n} \cdot \boldsymbol{\sigma}$, where $\mathbf{n}$ is a unit vector and $\boldsymbol{\sigma}$ are Pauli matrices. One crucial point is that quantum entanglement needs information of the reduced density matrix of a system, but the Bell's inequality does not. At first glance, this suggests that a quantitative entanglement measurement by the Bell's inequality is difficult. Thus, bridging the maximum violation of the Bell's inequality and measures of 
quantum entanglement provides a huge application of an entanglement measure without a bipartition to detect entanglement quantities.

There are various $n$-qubit systems exhibiting topological properties such as the toric code model [9] and the Wen-Plaquette model [10]. One of the topological signature is that the total quantum dimension of quasi-particles could be detected from the universal term in the entanglement entropy [11, 12], i.e., topological entanglement entropy $[13,14]$. This motivates us to apply our theorem to the Wen-Plaquette model. We find that the upper bound of the maximum violation of the Bell's inequality in the Wen-Plaquette model indicates that the ground state is maximally entangled. The use of the maximally entangled property for a six-qubit state in the Wen-Plaquette model could be related to the topological entanglement entropy via the maximum violation of the Bell's inequality.

\section{Entanglement and maximum violation}

A Bell's operator of $n$ qubits is defined iteratively as $\mathcal{B}_{n}[8]: \mathcal{B}_{n}=\mathcal{B}_{n-1} \otimes \frac{1}{2}\left(A_{n}+A_{n}^{\prime}\right)+$ $\mathcal{B}_{n-1}^{\prime} \otimes \frac{1}{2}\left(A_{n}-A_{n}^{\prime}\right)$, where $A_{n}=\mathbf{a}_{n} \cdot \boldsymbol{\sigma}$ and $A_{n}^{\prime}=\mathbf{a}_{n}^{\prime} \cdot \boldsymbol{\sigma}$ are the operators in the $n$-th qubit with $\mathbf{a}_{n}$ and $\mathbf{a}_{n}^{\prime}$ being unit vectors and $\boldsymbol{\sigma}=\left(\sigma_{x}, \sigma_{y}, \sigma_{z}\right)$ being the Pauli matrices. The operators $\frac{1}{2} \mathcal{B}_{n-1}$ and $\frac{1}{2} \mathcal{B}_{n-1}^{\prime}$ act on the rest of the qubits. Notice that we choose $\frac{1}{2} \mathcal{B}_{1}=\mathbf{b} \cdot \boldsymbol{\sigma}$ and $\frac{1}{2} \mathcal{B}_{1}^{\prime}=\mathbf{b}^{\prime} \cdot \boldsymbol{\sigma}$ with $\mathbf{b}$ and $\mathbf{b}^{\prime}$ being unit vectors. It is known that for an $n$-qubit system, the upper bound of the expectation value of the Bell's operator $\operatorname{Tr}\left(\rho \mathcal{B}_{n}\right) \leq 2^{\frac{n+1}{2}}$ [8] leads to the violation of the Bell-CHSH inequality [2].

For a given density matrix $\rho$, the maximum expectation value of a Bell's operator is referred to as the maximum violation of the Bell's inequality. Here we demonstrate a relation between the maximum violation of the Bell's inequality and a concurrence of a pure state (an entanglement quantity) in an $n$-qubit system when the all $i$-th operators in the Bell's operator are $A_{i}$ and $A_{i}^{\prime}$ for $2 \leq i<n$ :

$$
\begin{aligned}
\tilde{\mathcal{B}}_{n}= & \mathcal{B}_{1} \otimes A_{2} \otimes A_{3} \cdots \otimes A_{n-2} \otimes A_{n-1} \otimes \frac{1}{2}\left(A_{n}+A_{n}^{\prime}\right) \\
& +\mathcal{B}_{1}^{\prime} \otimes A_{2}^{\prime} \otimes A_{3}^{\prime} \cdots \otimes A_{n-2}^{\prime} \otimes A_{n-1}^{\prime} \otimes \frac{1}{2}\left(A_{n}-A_{n}^{\prime}\right) .
\end{aligned}
$$

To proceed our derivation, we introduce an $R$-matrix: $R_{i_{1} i_{2} \cdots i_{n}} \equiv \operatorname{Tr}\left(\rho \sigma_{i_{1}} \otimes \sigma_{i_{2}} \otimes \cdots \otimes\right.$ $\left.\sigma_{i_{n}}\right) \equiv R_{I i_{n}}$, where $\rho$ is a density matrix, $\sigma_{i_{\alpha}}$ is the Pauli matrix with $i_{\alpha}=x, y, z$ and $\alpha=1,2, \cdots, n$ are the site indices. We express the $R$-matrix as a $3^{n-1} \times 3$ matrix $R_{I i_{n}}$ with the first index being $I=i_{1} i_{2} \cdots i_{n-1}$ and the second index being $i_{n}$. In a two-qubit system, the maximum violation of the Bell's inequality is computed from a $3 \times 3$ matrix $R_{i j}$ defined above [7]. Now we generalize the maximum violation of the Bell's inequality $\left(\tilde{\mathcal{B}}_{n}\right)$ in an $n$-qubit system by using the $R$-matrix.

Lemma 1. The maximum violation of the Bell's inequalities $\gamma \equiv \max _{\tilde{\mathcal{B}}_{n}} \operatorname{Tr}\left(\rho \tilde{\mathcal{B}}_{n}\right) \leq$ $2 \sqrt{u_{1}^{2}+u_{2}^{2}}$, where $u_{1}^{2}$ and $u_{2}^{2}$ are the first two largest eigenvalues of $R^{\dagger} R$ when $n>2$ and $\gamma=2 \sqrt{u_{1}^{2}+u_{2}^{2}}$ when $n=2$. 
Proof. We first introduce two three-dimensional orthonormal vectors $\hat{c}$ and $\hat{c}^{\prime}$ such that $\hat{a}+\hat{a}^{\prime}=2 \hat{c} \cos \theta$ and $\hat{a}-\hat{a}^{\prime}=2 \hat{c}^{\prime} \sin \theta$, where $\theta \in\left[0, \frac{1}{2} \pi\right]$, through three-dimensional unit vectors $\hat{a}$ and $\hat{a}^{\prime}$. The maximum violation of the Bell's inequality is defined as $\gamma \equiv$ $\max _{\tilde{\mathcal{B}}_{n}} \operatorname{Tr}\left(\rho \tilde{\mathcal{B}}_{n}\right)$ with the Bell's operator of the $n$-qubit $\tilde{\mathcal{B}}_{n}$ defined in (2.1). By using the $R$-matrix and the unit vectors $\hat{B} \equiv \hat{B}_{I}=\hat{B}_{i_{1} i_{2} \cdots i_{n-1}} \equiv \hat{a}_{1, i_{1}} \hat{a}_{2, i_{2}} \cdots \hat{a}_{n-1, i_{n-1}}, \hat{B}^{\prime} \equiv \hat{B}_{I}^{\prime}=$ $\hat{B}_{i_{1} i_{2} \cdots i_{n-1}}^{\prime} \equiv \hat{a}_{1, i_{1}}^{\prime}{\hat{a^{\prime}}}_{2, i_{2}} \cdots{\hat{a^{\prime}}}_{n-1, i_{n-1}}, \hat{a} \equiv \hat{a}_{n, i_{n}}$, and ${\hat{a^{\prime}}}^{\prime} \equiv \hat{a}_{n, i_{n}}^{\prime}$, in which $\hat{B}$ and $\hat{B}^{\prime}$ are unit vectors in $3^{n-1}$ dimensions, we have $\gamma=\max _{\hat{B}, \hat{B}^{\prime}, \hat{a}, \hat{a^{\prime}}}\left(\left\langle\hat{B}, R\left(\hat{a}+\hat{a}^{\prime}\right)\right\rangle+\left\langle\hat{B}^{\prime}, R\left(\hat{a}-\hat{a}^{\prime}\right)\right\rangle\right) \leq$ $\max _{\hat{c}, \hat{c}^{\prime}, \theta}\left(2\|R \hat{c}\| \cos \theta+2\left\|R \hat{c^{\prime}}\right\| \sin \theta\right)=2 \sqrt{u_{1}^{2}+u_{2}^{2}}$, in which $u_{1}^{2}$ and $u_{2}^{2}$ are the first two largest eigenvalues of $R^{\dagger} R$. The inner product and the norm are defined as $\langle P, Q\rangle \equiv P^{\dagger} Q$ and $\|U\| \equiv \sqrt{U^{\dagger} U}$. Because $R\left(\hat{a}+\hat{a^{\prime}}\right)$ and $R\left(\hat{a}-\hat{a}^{\prime}\right)$ are defined in the $3^{n-1}$ dimensions and each unit vector $\hat{B}$ and $\hat{B}^{\prime}$ only contains $2(n-1)$ parameters, it could not guarantee that $\hat{B}$ parallels $R\left(\hat{a}+\hat{a}^{\prime}\right)$ and $\hat{B}^{\prime}$ parallels $R\left(\hat{a}-\hat{a}^{\prime}\right)$, except for $n=2$.

An earlier approach to relate the maximum violation of the Bell's inequality and the concurrence of a pure state $C(\psi) \equiv \sqrt{2\left(1-\operatorname{Tr} \rho_{A}^{2}\right)}[5]$ in a two-qubit system is discussed in [6].

We generalize the relation of the maximum violation of the Bell's inequality and the concurrence of a pure state in an $n$-qubit system when a state is a linear combination of two product states. The concurrence of a pure state is computed with respect to the bipartition with $(n-1)$ qubits in subsystem $B$ and one qubit in subsystem $A$. Here we emphasize that the generalization of the relation of the maximum violation of the Bell's inequality and the concurrence of an arbitraty pure state in an $n$-qubit system is extremely difficult. This specific state we considered provides one concrete example of the $n$-qubits generalization.

Theorem 1. For an n-qubit state $|\psi\rangle=|u\rangle_{B} \otimes\left(\lambda_{+}|v\rangle_{B} \otimes|1\rangle_{A}+\lambda_{-}|\tilde{v}\rangle_{B} \otimes|0\rangle_{A}\right)$ with $\lambda_{+}|v\rangle_{B} \otimes|1\rangle_{A}+\lambda_{-}|\tilde{v}\rangle_{B} \otimes|0\rangle_{A}$ being a non-biseparable $\alpha$-qubit state, $|u\rangle_{B},|v\rangle_{B},|\tilde{v}\rangle_{B}$ being product states consisting of $|0\rangle$ 's and $|1\rangle$ 's, $|v\rangle_{B}$ and $|\tilde{v}\rangle_{B}$ being orthogonal, and the maximum violation of the Bell's inequality in an $n$-qubit system is $\gamma=2 f_{\alpha}(\psi)$, in which the function $f_{\alpha}(\psi)$ is defined as:

(1) $\alpha$ is an even number:

$$
\begin{array}{rlrl}
f_{\alpha}(\psi) & \equiv \sqrt{1+2^{\alpha-2} C^{2}(\psi)}, & 2^{2-\alpha} \geq C^{2}(\psi), \\
f_{\alpha}(\psi) \equiv 2^{\frac{\alpha-1}{2}} C(\psi), & 2^{2-\alpha} \leq C^{2}(\psi) .
\end{array}
$$

(2) $\alpha$ is an odd number:

$$
\begin{array}{lll}
f_{\alpha}(\psi) \equiv \sqrt{1+\left(2^{\alpha-2}-1\right) C^{2}(\psi)}, & \frac{1}{1+2^{\alpha-2}} \geq C^{2}(\psi), \\
f_{\alpha}(\psi) \equiv 2^{\frac{\alpha-1}{2}} C(\psi), & \frac{1}{1+2^{\alpha-2}} \leq C^{2}(\psi) .
\end{array}
$$

Here, $C(\psi)$ is the purity of a pure state computed with respect to the bipartition that subsystem $B$ contains $(n-1)$ qubits and subsystem A contains one qubit. 
Proof. The Hilbert space for an $n$-qubit system is bipartitioned as $\mathbb{H}=\mathbb{H}_{B} \otimes \mathbb{H}_{A}$, in which dimensions of the sub-Hilbert spaces are $\operatorname{dim}\left(\mathbb{H}_{A}\right)=2$ and $\operatorname{dim}\left(\mathbb{H}_{B}\right)=2^{n-1}$. We consider a pure state with respect to this bipartition $|\psi\rangle=|u\rangle_{B} \otimes\left(\lambda_{+}|v\rangle_{B} \otimes|1\rangle_{A}+\lambda_{-}|\tilde{v}\rangle_{B} \otimes|0\rangle_{A}\right)$, where $|u\rangle_{B} \otimes|v\rangle_{B}$ and $|u\rangle_{B} \otimes|\tilde{v}\rangle_{B}$ are the product states in $\mathbb{H}_{B}$ and $|1\rangle_{A}$ and $|0\rangle_{A}$ are the states in $\mathbb{H}_{A}$. By using the property $\operatorname{Tr} \rho_{A}=\lambda_{+}^{2}+\lambda_{-}^{2}=1$ and $C(\psi)=\sqrt{2\left(1-\lambda_{+}^{4}-\lambda_{-}^{4}\right)}$, the coefficients $\lambda_{ \pm}$can be expressed in terms of the concurrence of the pure state, $\lambda_{ \pm}^{2}=$ $\left(1 \pm \sqrt{1-C^{2}(\psi)}\right) / 2$. The matrix elements of the $R$-matrix are

$$
\begin{aligned}
& R_{I x}=\lambda_{+} \lambda_{-} \operatorname{Tr}\left[\sigma_{I_{1}}|u\rangle\langle u| \otimes \sigma_{I_{2}}(|v\rangle\langle\tilde{v}|+| \tilde{v}\rangle\langle v|)\right], \\
& R_{I y}=-i \lambda_{+} \lambda_{-} \operatorname{Tr}\left[\sigma_{I_{1}}|u\rangle\langle u| \otimes \sigma_{I_{2}}(|v\rangle\langle\tilde{v}|-| \tilde{v}\rangle\langle v|)\right], \\
& R_{I z}=-\lambda_{+}^{2} \operatorname{Tr}\left[\sigma_{I_{1}}|u\rangle\left\langle u\left|\otimes \sigma_{I_{2}}\right| v\right\rangle\langle v|\right]+\lambda_{-}^{2} \operatorname{Tr}\left[\sigma_{I_{1}}|u\rangle\left\langle u\left|\otimes \sigma_{I_{2}}\right| \tilde{v}\right\rangle\langle\tilde{v}|\right],
\end{aligned}
$$

where $I \equiv I_{1} I_{2}$ concatenating two strings of indices $I_{1} \equiv i_{1} \cdots i_{n-\alpha-1}$ and $I_{2} \equiv i_{n-\alpha} \cdots i_{n-1}$, and $\sigma_{I 1} \equiv \sigma_{i_{1}} \otimes \cdots \otimes \sigma_{i_{n-\alpha-1}}, \sigma_{I 2} \equiv \sigma_{i_{n-\alpha}} \otimes \cdots \otimes \sigma_{i_{n-1}}$. Here we choose the basis that $|0\rangle \equiv(1,0)^{\mathrm{T}}$ and $|1\rangle \equiv(0,1)^{\mathrm{T}}$. One should notice that the dimensions of $|u\rangle\langle u|$ is $2^{n-\alpha}$, and the dimensions of $|v\rangle\langle v|,| \tilde{v}\rangle\langle\tilde{v}|| v\rangle,\langle\tilde{v}|$, and $|\tilde{v}\rangle\langle v|$ are $2^{\alpha-1}$. The nonvanishing matrix elements of $R_{I \alpha}, \alpha=x, y, z$, come from the diagonal matrix elements of $\sigma_{I_{1}}|u\rangle\left\langle u\left|\otimes \sigma_{I_{2}}\right| v\right\rangle\left\langle v\left|, \sigma_{I_{1}}\right| u\right\rangle\left\langle u\left|\otimes \sigma_{I_{2}}\right| \tilde{v}\right\rangle\left\langle\tilde{v}\left|, \sigma_{I_{1}}\right| u\right\rangle\left\langle u\left|\otimes \sigma_{I_{2}}\right| \tilde{v}\right\rangle\langle v|$ and $\sigma_{I_{1}}|u\rangle\left\langle u\left|\otimes \sigma_{I_{2}}\right| v\right\rangle\langle\tilde{v}|$. Then the conditions of non-vanishing matrix elements of $R_{I \alpha}$, where $\alpha=x, y, z$, require that $\sigma_{I_{1}}|u\rangle \rightarrow|u\rangle, \sigma_{I_{2}}|\tilde{v}\rangle \rightarrow|v\rangle$ and $\sigma_{I_{2}}|v\rangle \rightarrow|\tilde{v}\rangle$ for $R_{I x(y)}$ and $\sigma_{I_{2}}|u\rangle \rightarrow|u\rangle, \sigma_{I_{2}}|v\rangle \rightarrow|v\rangle$ and $\sigma_{I_{2}}|\tilde{v}\rangle \rightarrow|\tilde{v}\rangle$ for $R_{I z}$.

The conditions for non-vanishing matrix elements $R_{I x}$ are $(n-\alpha)$ number of $\sigma_{z}$ matrices in $I_{1},(\alpha-1-i)$ number of $\sigma_{x}$ matrices and $i$ number of $\sigma_{y}$ matrices in $I_{2}$ with $i$ being an even integer. The conditions for non-vanishing matrix elements $R_{I y}$ are $(n-\alpha)$ number of $\sigma_{z}$ matrices in $I_{1},(\alpha-1-j)$ number of $\sigma_{x}$ matrices and $j$ number of $\sigma_{y}$ matrices in $I_{2}$ with $j$ being an odd integer. The conditions for non-vanishing matrix elements $R_{I z}$ are $(n-\alpha)$ number of $\sigma_{z}$ matrices in $I_{1},(\alpha-1)$ number of $\sigma_{z}$ matrices in $I_{2}$.

The above conditions lead to the diagonal form of the matrix $R^{\dagger} R$. In the case that $\alpha$ is an even integer, the set of eigenvalues of $R^{\dagger} R$ is $\left\{2^{\alpha-2} C^{2}(\psi), 2^{\alpha-2} C^{2}(\psi), 1\right\}$. In the case that $\alpha$ is an odd integer, the set of eigenvalues of $R^{\dagger} R$ is $\left\{2^{\alpha-2} C^{2}(\psi), 2^{\alpha-2} C^{2}(\psi), 1-C^{2}(\psi)\right\}$.

Now we show $\gamma=\max _{\hat{B}, \hat{B}^{\prime}, \hat{a}, \hat{a}^{\prime}}\left\langle\hat{B}, R\left(\hat{a}+\hat{a}^{\prime}\right)\right\rangle+\left\langle\hat{B}^{\prime}, R\left(\hat{a}-\hat{a}^{\prime}\right)\right\rangle=2 \sqrt{u_{1}^{2}+u_{2}^{2}}$, in which $u_{1}^{2}$ and $u_{2}^{2}$ are the first two largest eigenvalues of $R^{\dagger} R, \hat{B} \equiv \hat{a}_{1, i_{1}} \hat{a}_{2, i_{2}} \cdots \hat{a}_{n-1, i_{n-1}}$, $\hat{B}^{\prime} \equiv{\hat{a^{\prime}}}_{1, i_{1}}{\hat{a^{\prime}}}_{2, i_{2}} \cdots{\hat{a^{\prime}}}_{n-1, i_{n-1}}, \hat{a} \equiv \hat{a}_{n, i_{n}}$ and ${\hat{a^{\prime}}}^{\prime} \equiv{\hat{a^{\prime}}}_{n, i_{n}}^{\prime}$, where $\hat{a}_{n, i_{n}}+{\hat{a^{\prime}}}_{n, i_{n}} \equiv 2 \hat{c}_{n, i_{n}} \cos \theta$, $\hat{a}_{n, i_{n}}-{\hat{a^{\prime}}}_{n, i_{n}} \equiv 2 \hat{c}_{n, i_{n}} \sin \theta$, and $\theta \in[0, \pi / 2]$. This equality holds when $\hat{B}$ parallels $R\left(\hat{a}+\hat{a}^{\prime}\right)$ and $\hat{B}^{\prime}$ parallels $R\left(\hat{a}-\hat{a}^{\prime}\right)$. One natural choice of $a_{\alpha, i_{\alpha}}$ and $a_{\alpha, i_{\alpha}}$ could be obtained by equating two ratios, $\left|R_{I x}\left(\hat{a}_{x}+\hat{a}_{x}^{\prime}\right) / R_{I^{\prime} y}\left(\hat{a}_{y}+\hat{a}_{y}^{\prime}\right)\right|=\left|B_{I} / B_{I^{\prime}}\right|$ and $\left|R_{I x}\left(\hat{a}_{x}-\hat{a}_{x}^{\prime}\right) / R_{I^{\prime} y}\left(\hat{a}_{y}-\hat{a}_{y}^{\prime}\right)\right|=$ $\left|B_{I}^{\prime} / B_{I^{\prime}}^{\prime}\right|$, where $I$ and $I^{\prime}$ is chosen in a way that one site of the $I_{2}$ in $I$ is labeled by $x$ and in $I^{\prime}$ is labeled by $y$, and other sites of the $I_{2}$ in $I$ and $I^{\prime}$ are labeled by the same symbols. This leads to $\left|\hat{a}_{I, x} / \hat{a}_{I^{\prime}, y}\right|=\left|\hat{c}_{n, x} / \hat{c}_{n, y}\right|$ and $\left|\hat{a}_{I, x}^{\prime} /{\hat{a^{\prime}}}_{I^{\prime}, y}\right|=\left|\hat{c}_{n, x}^{\prime} / \hat{c}_{n, y}^{\prime}\right|$. When $u_{1}^{2}=\left(R^{\dagger} R\right)_{x x}$ and $u_{2}^{2}=\left(R^{\dagger} R\right)_{y y}$, we choose $\left(\hat{c}_{n, x}, \hat{c}_{n, y}, \hat{c}_{n, z}\right)^{\mathrm{T}}=(1 / \sqrt{2})(1,1,0)^{\mathrm{T}},\left(\hat{c}_{n, x}^{\prime}, \hat{c}^{\prime}{ }_{n, y}, \hat{c}^{\prime}{ }_{n, z}\right)^{\mathrm{T}}=$ $(1 / \sqrt{2})(1,-1,0)^{\mathrm{T}}$, and $\cos (\theta)=\sin (\theta)=\sqrt{2} / 2$ to show $\gamma=2 \sqrt{u_{1}^{2}+u_{2}^{2}}$. For the other case $u_{1}^{2}=\left(R^{\dagger} R\right)_{z z}$ and $u_{2}^{2}=\left(R^{\dagger} R\right)_{x x}$, we could choose $\left(\hat{c}_{n, x}, \hat{c}_{n, y}, \hat{c}_{n, z}\right)^{\mathrm{T}}=(0,0,1)^{\mathrm{T}}$, 
$\left({\hat{c^{\prime}}}_{n, x},{\hat{c^{\prime}}}_{n, y},{\hat{c^{\prime}}}_{n, z}\right)^{\mathrm{T}}=(1 / \sqrt{2})(1,1,0)^{\mathrm{T}}, \cos (\theta)=u_{1} / \sqrt{u_{1}^{2}+u_{2}^{2}}$ and $\sin (\theta)=u_{2} / \sqrt{u_{1}^{2}+u_{2}^{2}}$ to prove $\gamma=2 \sqrt{u_{1}^{2}+u_{2}^{2}}$.

We demonstrate that the maximum violation of the Bell's inequality $\left(\tilde{\mathcal{B}}_{n}\right)$ is directly related to the concurrence of the pure state when the subsystem $A$ only contains one qubit and the state is a linear combination of two product states. The maximally entangled state we considered is a bipartite pure state, in which the reduced density matrix has the diagonal form $\rho_{A}=\sum_{\nu=1}^{n} \frac{1}{n}|\nu\rangle\langle\nu|$ with $\nu$ is the state in a subsystem $A$. Since the subsystem $A$ contains only one site, the reduced density matrix of the maximally entangled state has the diagonal form $\rho_{A}=\frac{1}{2}(|0\rangle\langle 0|+| 1\rangle\langle 1|)$. The concurrence of this maximally entangled state equals to one $C(\psi)=1$, which leads to the maximum violation of the Bell's inequality $\gamma=2^{\frac{\alpha+1}{2}} \leq 2^{\frac{n+1}{2}}$ satisfies the upper bound of the Bell's operator of an $n$-qubit system. Although we do not use the most generic form of the Bell's operator, information of the state could be complete when the $n$-th qubit operators are measured. The extrapolation of the maximum violation of the Bell's inequality $\left(\tilde{\mathcal{B}}_{n}\right)$ from the $R$-matrix could be equivalent to direct computing of the maximum violation of the Bell's inequality without losing generality.

It is interesting to note that the state could reach the upper bound of the Bell's inequality $\left(\mathcal{B}_{n}\right)$ even if we consider the simplified Bell's operator $\left(\tilde{\mathcal{B}}_{n}\right)$. A generic state in this simplified Bell's operator is still hard to get an exact solution so we demonstrate the relation between the simplified Bell's operator and the entanglement quantities in the state, which is a linear combination of two product states and is similar to the case of two-qubit.

\section{Applications to the Wen-Plaquette model}

The Wen-Plaquette model [10] is defined by the Hamiltonian on a two-dimensional periodic lattice (torus) as $H=\sum_{i} \sigma_{x}^{i} \sigma_{y}^{i+\hat{x}} \sigma_{x}^{i+\hat{x}+\hat{y}} \sigma_{y}^{i+\hat{y}}$, in which qubits live on the vertices with the four-spin interaction on each plaquette. A ground state of the Hamiltonian is an $n$-qubit state with $n$ being a number of vertices. We first apply our Theorem to a four-qubit state, with the geometry of the system containing four vertices, eight edges, and four faces. ${ }^{1}$ There are four degenerate ground states $|G\rangle_{4-\text { qubit }}=\frac{1}{\sqrt{2}}(|0000\rangle+|1111\rangle), \frac{1}{\sqrt{2}}(|1010\rangle+|0101\rangle)$, $\frac{1}{\sqrt{2}}(|0011\rangle-|1100\rangle), \frac{1}{\sqrt{2}}(|1001\rangle-|0110\rangle)$. The order of each site in these four-qubit states are defined in the figure 1 (a). Since the maximum violation of the Bell's inequalities for these ground states are $\gamma=4 \sqrt{2}$, the ground states have the maximum concurrence of the pure state $C(\psi)=1$ according to the Theorem and are maximally entangled.

Before computing the upper bound of the maximum violation of the Bell's inequality of a ground state of the six-qubit in the Wen-Plaquette model, we consider the six-qubit state, $|G\rangle_{6-\text { qubit }}=\frac{\lambda_{+}}{\sqrt{2}}(-|111000\rangle+|001110\rangle)+\frac{\lambda_{-}}{\sqrt{2}}(|100011\rangle+|010101\rangle)$, with the site labels shown in figure 1 (b). We relate the upper bound of the maximum violation of the Bell's inequality to the concurrence of the state. Two different bipartitions are considered:

\footnotetext{
${ }^{1}$ The Euler number of the torus is zero, $\chi=V-E+F=0$ with $V, E$, and $F$ being the number of vertices, edges, and faces, respectively.
} 
(a)

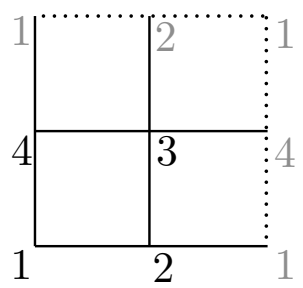

(b)

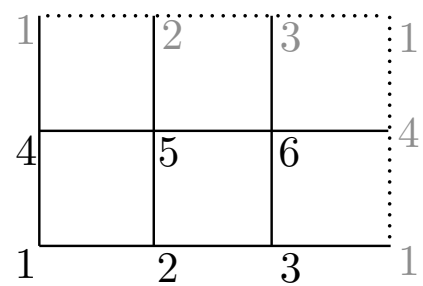

Figure 1. (a) A four-qubit state and (b) A six-qubit state for the Wen-Plaquette model on a torus. The right dashed line is identified as the left solid line and the top dashed line is identified as the bottom solid line in (a) and (b). The numbers are the site indices. Each gray colored number is identified with the corresponding black colored number.

(1) subsystem $A$ contains site number six, and (2) subsystem $A$ contains sites number five and number six. Here we use $\delta=1$ or 2 as an indicator for the case one and the case two. According to the Lemma, we find that the upper bound of the maximum violation of the Bell's inequality could be expressed as a function of the concurrence of the pure state when we exchange the final site with the first site in the Bell's operator $\left(\tilde{\mathcal{B}}_{n}\right)$ (We define $C(\delta) \equiv \sqrt{2\left(1-2^{\delta-1} \operatorname{Tr} \rho_{A(\delta)}^{2}\right)}$ as the generalized concurrence of the pure state for the sixqubit state with respect to two different bipartitions. This generalized concurrence has the property that $C(\delta)=1$ in a region $A$ indicates that the state is maximally entangled in a region $A$.) (see appendix $\mathrm{A}$ ).

In the case that $\lambda_{+}=\lambda_{-}=1 / \sqrt{2}$, the six-qubit state is a ground state of the WenPlaquette model and has the maximum generalized concurrence of the pure state $C(\delta)=1$ in the subregion, which indicates the maximally entangled state in the subregion. The entanglement entropy with respect to the two bipartitions are $S_{A(\delta=1)}=\ln 2$ and $S_{A(\delta=2)}=$ $2 \ln 2$, which could be obtained from the $R$-matrix through the inverse mapping, $\gamma \leq 6=$ $2 \sqrt{13-2^{\delta+2} e^{-S_{A(\delta)}}}$. We also find that the upper bound of the maximum violation of Bell's inequality $(\tilde{\mathcal{B}})$ given by our Lemma increases with respect to the generalized concurrence of the pure state as in the case of two qubits.

In general, the entanglement entropy has the form $S_{A(L)}=\alpha L-S_{\mathrm{TEE}}$, in which the first term indicates the area law with $L$ being the length of the entangling boundary, $\alpha$ being a constant, and $S_{\text {TEE }}$ is called the topological entanglement entropy. In the Wen-Plaquette model, the length of an entangling boundary $L$ is the number of bonds that connect a subsystem $A$ and a subsystem $B$. The entanglement entropy is a linear function of $L$ with two unknown variables $\alpha$ and $S_{\text {TEE }}$. Once the entanglement entropy $S_{A}$ with respect to two different length of entangling boundaries are computed, the topological entanglement entropy $S_{\text {TEE }}$ is obtained. We consider $L(\delta=1)=4$ and $L(\delta=2)=6$ to extract the area law of the entanglement entropy and obtain the topological entanglement entropy, $S_{\text {TEE }}=\ln 2=\ln \sqrt{D}$, where $D=4$ is the number of distinct quasiparticles $[13,14]$. Here, we demonstrate an indirect measurement of the topological entanglement entropy by measuring the $R$-matrix. 


\section{Outlook}

Recently, the ground states in the toric code model with three sites have realized in [15] by using a ${ }^{13} \mathrm{C}$-labeled trichloroethylene molecule. The ground states in the Wen-Plaquette model with four sites were also measured in the Iodotrifluroethylene $\left(\mathrm{C}_{2} \mathrm{~F}_{3} \mathrm{I}\right)[16,17]$ by using geometric algebra procedures [18], which could give a four-body interaction [16, 17] from the combination of two-body interactions and radio-frequency pulses [18, 19]. These systems provide natural platforms for testifying our theoretical studies.

\section{Acknowledgments}

We would like to thank Ling-Yan Hung and Xueda Wen for their insightful discussion. C.-T. M would like to thank Nan-Peng Ma for his encouragement. P.-Y. C. was supported by the Rutgers Center for Materials Theory. S.-K. C. was supported by the AFOSR, NSF QIS, ARL CDQI, ARO MURI, ARO and NSF PFC at JQI.

\section{A Maximum violation of the six-qubit state}

The density matrix is

$$
\begin{aligned}
\rho=|\psi\rangle\langle\psi|= & \frac{\lambda_{+}^{2}}{2}(|111000\rangle\langle 111000|+| 001110\rangle\langle 001110| \\
& -|111000\rangle\langle 001110|-| 001110\rangle\langle 111000|) \\
& +\frac{\lambda_{-}^{2}}{2}(|100011\rangle\langle 100011|+| 010101\rangle\langle 010101| \\
& +|100011\rangle\langle 010101|+| 010101\rangle\langle 100011|) \\
& +\frac{\lambda_{+} \lambda_{-}}{2}(-|111000\rangle\langle 100011|-| 111000\rangle\langle 010101| \\
& +|001110\rangle\langle 100011|+| 001110\rangle\langle 010101| \\
& -|100011\rangle\langle 111000|-| 010101\rangle\langle 111000| \\
& +|100011\rangle\langle 100011|+| 010101\rangle\langle 001110|) .
\end{aligned}
$$

The non-vanishing matrix elements of the $R$-matrix are

$$
\begin{array}{ll}
R_{z z z z z z}=R_{y y z x x z}=R_{x x z y y z}=-1, \\
R_{x x z x x z}=R_{y x z y x z}=R_{x y z x y z}=R_{y y z y y z}=\lambda_{+}^{2}-\lambda_{-}^{2} \\
R_{y x z x y z}=R_{x y z y x z}=1, \\
R_{z y y z x x}=-2 \lambda_{+} \lambda_{-}, \quad R_{z x y z y x}=2 \lambda_{+} \lambda_{-} \\
R_{y z y x z x}=-2 \lambda_{+} \lambda_{-}, \quad R_{x z y y z x}=2 \lambda_{+} \lambda_{-} \\
R_{z y x z x y}=2 \lambda_{+} \lambda_{-}, \quad R_{z x x y y y}=-2 \lambda_{+} \lambda_{-}, \\
R_{y z x x z y}=2 \lambda_{+} \lambda_{-}, \quad R_{x z x y z y}=-2 \lambda_{+} \lambda_{-} .
\end{array}
$$

The eigenvalues of $R^{\dagger} R$ are

$$
5+4\left(\lambda_{+}^{2}-\lambda_{-}^{2}\right)^{2}, \quad 16 \lambda_{+}^{2} \lambda_{-}^{2}, \quad 16 \lambda_{+}^{2} \lambda_{-}^{2} .
$$


If we denote the last qubit as the region $A$ and the complementary region as the region $B$, the entanglement entropy of the region $A$ is

$$
-\lambda_{+}^{2} \ln \lambda_{+}^{2}-\lambda_{-}^{2} \ln \lambda_{-}^{2} .
$$

We also obtain

$$
\operatorname{Tr} \rho_{A}^{2}=\lambda_{+}^{4}+\lambda_{-}^{4}=1-2 \lambda_{+}^{2} \lambda_{-}^{2} .
$$

Therefore, this could imply

$$
\lambda_{+}^{2}=\frac{1 \pm \sqrt{1-C^{2}(1, \psi)}}{2}, \quad \lambda_{-}^{2}=\frac{1 \mp \sqrt{1-C^{2}(1, \psi)}}{2} .
$$

It is easy find that the entanglement entropy of the region $A$ could increase by increasing the concurrence $C(1, \psi)$. Therefore, the eigenvalues of $R^{\dagger} R$ are

$$
9-4 C^{2}(1, \psi), \quad 4 C^{2}(1, \psi), \quad 4 C^{2}(1, \psi) .
$$

The maximum violation of the Bell's inequality is

$$
\gamma \leq 6 .
$$

If we denote the last two qubits as the region $A$ and the complementary region as the region $B$, the entanglement entropy of the region $A$ is

$$
\ln 2-\lambda_{+}^{2} \ln \lambda_{+}^{2}-\lambda_{-}^{2} \ln \lambda_{-}^{2} .
$$

We also have

$$
\operatorname{Tr} \rho_{A}^{2}=\frac{\lambda_{+}^{4}}{2}+\frac{\lambda_{-}^{4}}{2}=\frac{1}{2}-\lambda_{+}^{2} \lambda_{-}^{2} .
$$

Thus, we could show

$$
\lambda_{+}^{2}=\frac{1 \pm \sqrt{1-C^{2}(2, \psi)}}{2}, \quad \lambda_{-}^{2}=\frac{1 \mp \sqrt{1-C^{2}(2, \psi)}}{2} .
$$

It is easy find that the entanglement entropy of the region $A$ could monotonically increase by increasing the parity $C(2, \psi)$. Therefore, the eigenvalues of $R^{\dagger} R$ are

$$
9-4 C^{2}(2, \psi), \quad 4 C^{2}(2, \psi), \quad 4 C^{2}(2, \psi) .
$$

The maximum violation of the Bell's inequality is

$$
\gamma \leq 6
$$

The above result shows that the upper bound of the maximum violation of the Bell's inequality does not depend on the parity of the pure state. To relate the upper bound of 
the maximum violation of the Bell's inequality to the parity of the pure state, we exchange the final site with the first site for the Bell's operator $(\tilde{\mathcal{B}})$. Thus, we could find that the eigenvalues of $R^{\dagger} R$ are

$$
1+4 C^{2}(1, \psi)=1+4 C^{2}(2, \psi), \quad 4, \quad 4 .
$$

When $C^{2}(1, \psi)=C^{2}(2, \psi) \geq 3 / 4$, the maximum violation of the Bell's inequality is

$$
\gamma \leq 2 \sqrt{5+4 C^{2}(1, \psi)}=2 \sqrt{5+4 C^{2}(2, \psi)} .
$$

When $C^{2}(1, \psi)=C^{2}(2, \psi)<3 / 4$, the maximum violation of the Bell's inequality is

$$
\gamma \leq 4 \sqrt{2}
$$

Open Access. This article is distributed under the terms of the Creative Commons Attribution License (CC-BY 4.0), which permits any use, distribution and reproduction in any medium, provided the original author(s) and source are credited.

\section{References}

[1] A. Steane, Quantum computing, Rept. Prog. Phys. 61 (1998) 117 [quant-ph/9708022] [INSPIRE].

[2] J.F. Clauser, M.A. Horne, A. Shimony and R.A. Holt, Proposed experiment to test local hidden variable theories, Phys. Rev. Lett. 23 (1969) 880 [INSPIRE].

[3] J.S. Bell, On the Einstein-Podolsky-Rosen paradox, Physics 1 (1964) 195 [INSPIRE].

[4] B.S. Cirelson, Quantum generalizations of Bell's inequality, Lett. Math. Phys. 4 (1980) 93 [INSPIRE].

[5] C.H. Bennett, D.P. DiVincenzo, J.A. Smolin and W.K. Wootters, Mixed state entanglement and quantum error correction, Phys. Rev. A 54 (1996) 3824 [quant-ph/9604024] [INSPIRE].

[6] F. Verstraete and M.M. Wolf, Entanglement versus Bell violations and their behaviour under local filtering operations, Phys. Rev. Lett. 89 (2002) 170401 [quant-ph/0112012].

[7] R. Horodecki, P. Horodecki and M. Horodecki, Violating Bell inequality by mixed spin-12 states: necessary and sufficient condition, Phys. Lett. A 200 (1995) 340.

[8] N. Gisin and H. Bechmann-Pasquinucci, Bell inequality, Bell states and maximally entangled states for $n$ qubits, Phys. Lett. A 246 (1998) 1 [quant-ph/9804045] [inSPIRE].

[9] A. Yu. Kitaev, Fault tolerant quantum computation by anyons, Annals Phys. 303 (2003) 2 [quant-ph/9707021] [INSPIRE].

[10] X.-G. Wen, Quantum orders in an exact soluble model, Phys. Rev. Lett. 90 (2003) 016803 [quant-ph/0205004] [INSPIRE].

[11] A. Hamma, R. Ionicioiu and P. Zanardi, Bipartite entanglement and entropic boundary law in lattice spin systems, Phys. Rev. A 71 (2005) 022315 [InSPIRE].

[12] L.-Y. Hung and Y. Wan, Revisiting Entanglement Entropy of Lattice Gauge Theories, JHEP 04 (2015) 122 [arXiv: 1501.04389] [INSPIRE]. 
[13] A. Kitaev and J. Preskill, Topological entanglement entropy, Phys. Rev. Lett. 96 (2006) 110404 [hep-th/0510092] [INSPIRE].

[14] T. Grover, Y. Zhang and A. Vishwanath, Entanglement Entropy as a Portal to the Physics of Quantum Spin Liquids, New J. Phys. 15 (2013) 025002 [arXiv:1302.0899] [InSPIRE].

[15] K. Li et al., Experimental Identification of Non-Abelian Topological Orders on a Quantum Simulator, Phys. Rev. Lett. 118 (2017) 080502 [arXiv: 1608. 06932].

[16] Z. Luo et al., Experimental Preparation of Topologically Ordered States via Adiabatic Evolution, arXiv:1608.06963 [arXiv:1608.06963].

[17] Z. Luo et al., Identifying Topological Order by Measuring the Modular Matrices, arXiv: 1608.06978 [arXiv:1608.06978].

[18] X. Peng, J. Zhang, J. Du and D. Suter, Quantum simulation of a system with competing twoand three-body interactions, Phys. Rev. Lett. 103 (2009) 140501 [arXiv:0809.0589] [arXiv: 0809.0989].

[19] C.H. Tseng et al., Quantum simulation of a three-body interaction Hamiltonian on an NMR quantum computer, Phys. Rev. A 61 (2000) 012302 [quant-ph/9908012]. 